\title{
Immobilized Talaromyces thermophilus lipase as an efficient catalyst for the production of LML-type structured lipids
}

\begin{abstract}
LML-type structured lipids are one type of medium- and long-chain triacylglycerols. LML was synthesized using immobilized Talaromyces thermophilus lipase (TTL)-catalyzed interesterification of tricaprylin and ethyl linoleate. The resin AB-8 was chosen, and the lipase/support ratio was determined to be $60 \mathrm{mg} / \mathrm{g}$. Subsequently, the immobilized TTL with strict sn-1,3 regiospecificity was applied to synthesize LML. Under the optimized conditions $\left(60{ }^{\circ} \mathrm{C}\right.$, reaction time $6 \mathrm{~h}$, enzyme loading of $6 \%$ of the total weight of substrates, substrate of molar ratio of ethyl linoleate to tricaprylin of 6:1), Triacylglycerols with two long- and one medium-chain FAs (DL-TAG) content as high as 52.86 mol\% was obtained. Scale-up reaction further verified the industrial potential of the established process. The final product contained 85.24 mol\% DL-TAG of which $97 \mathrm{~mol} \%$ was LML after purification. The final product obtained with the high LML content would have substantial potential to be used as functional oils.
\end{abstract}

Keyword: Talaromyces thermophilus lipase; Immobilization; Interesterification; Structured lipids; Functional oils 